\title{
EDITORIAL
}

\section{Counseling patients about obstetric risk: the breech experience}

\section{Journal of Perinatology (2007) 27, 139-140. doi:10.1038/sj.jp.7211671}

The cesarean delivery rate in the US has risen from $5.5 \%$ in 1970 to reach its highest level yet in 2005 of $30.2 \%$, ${ }^{1,2}$ despite a goal for the primary cesarean delivery rate from Healthy People 2010 of $15 \%{ }^{3}$ While the incidence of cesarean delivery is rapidly rising overall, its ascent has been much faster in two particular groups of women, those with a prior cesarean and those with a breech presentation. As a tactic to alleviate the rapid rise in cesarean delivery seen in the 1980s, a trial of labor after prior cesarean was actively encouraged in the early 1990s. ${ }^{4}$ At the same time, cesarean deliveries for breech presentation continued to increase throughout the 1990s, despite the availability of only small retrospective studies to examine perinatal outcomes from vaginal breech deliveries.

There are both similarities as well as some subtle differences in decision-making regarding mode of delivery when comparing women with a prior cesarean versus those with a breech presentation. In both cases, it appears from both retrospective and prospective data that planned cesarean delivery can lead to lower rates of neonatal morbidity and mortality, but that this difference is quite small at 1 in 500 to 1 in 1000. Another similarity is that in both cases, the immediate outcomes and recovery time for the pregnant women seem to favor a trial of labor. A clinical difference is that performing a cesarean on women with a breech presentation confers upon them the risk of having had a prior cesarean in future pregnancies, whereas this risk already exists for women with a prior cesarean delivery. Another difference, one that appears to contribute significantly to practice variations between the two scenarios, is that, in technical terms, a vaginal birth after cesarean is similar to other vaginal births, whereas a breech vaginal delivery requires different, highly skilled maneuvers requiring specific training. A recent ACOG Committee Opinion stated, 'Cesarean delivery will be the preferred mode of delivery for most physicians because of the diminishing expertise in vaginal breech delivery. ${ }^{5}$

With respect to breech presentation, there has been a flurry of activity examining outcomes related to planned mode of delivery in this setting over the past decade. The largest contributor is the Term Breech Trial (TBT) conducted by Hannah et al. ${ }^{6}$ In their initial publication of this prospective randomized trial, they demonstrated that short-term neonatal mortality and morbidity was higher in the group with planned vaginal delivery. However, in a 2-year follow-up study, they actually demonstrated no differences in morbidity and mortality outcomes between the two groups. ${ }^{7}$ To give further context, in France, where breech vaginal delivery is still commonly practiced, a large recent study found no clinically or statistically significant difference in either neonatal morbidity or mortality between planned vaginal delivery and planned cesarean delivery strategies. Unfortunately, in the US, the number of obstetricians comfortable with offering a vaginal delivery to women with a breech presentation appears to be falling rapidly, so it is unclear whether similar outcomes to the French study can be achieved in this country.

In the current issue of the Journal of Perinatology, there are two studies of mode of delivery in the setting of breech presentation. The first, by Hopkins et al., is a retrospective cohort study with similar findings to the recent French study. In a single institution, the authors demonstrate that with their protocol of routine CT pelvimetry, they find no clinically or statistically significant differences in severe morbidity or mortality. However, they do demonstrate higher rates of such markers as umbilical artery acidemia and admissions to the intensive care nursery in the neonates delivered vaginally. But these risks should be weighed against higher rates of infectious and bleeding morbidity in the mothers who underwent cesarean delivery. It appears from this and other recent studies, that counseling women with the evidence and facilitating their decision regarding mode of delivery, as is recommended by $\mathrm{ACOG}$, is the most reasonable approach.

In the other study by Lee et al., the authors demonstrate that in the US in 1999-2000, there were fewer cesarean deliveries among mothers of African-American race as compared to White women. This difference disappeared when socioeconomic factors such as education were controlled for, but a lower cesarean delivery rate between those women with lower education levels as compared to women with higher education levels persisted. If women are being well counseled, using culturally, linguistically and educationally appropriate approaches, it is possible that education level simply serves as a proxy for women's preferences towards mode of delivery. However, we must raise the possibility that women with lower socioeconomic status may be receiving counseling regarding mode of delivery, which fails to match their levels of health literacy or which is not presented in communication style that encourages informed participation for all populations. These latter two concerns are paramount for clinicians and policy-makers alike to examine to ensure equal quality of care to women of all educational, socioeconomic and racial/ethnic backgrounds.

While it was anticipated that the TBT would put the controversy surrounding the optimal management of breech presentation to 
rest, it appears that questions regarding the absolute risk to both mother and baby still persist. This might appear to be a minor issue at a time when cesarean delivery on maternal request is being considered for all parturients. ${ }^{8}$ However, the majority of women still prefer to achieve vaginal delivery ${ }^{9}$ and it appears that the majority of women may do so safely, even with a breech presentation. Thus, it is incumbent upon training institutions to train clinicians with the skills necessary to care for such women. Further, it is important for clinicians to counsel women regarding the options of care, including the long-term outcomes and effects on future pregnancies.

\section{$\mathrm{AB}$ Caughey ${ }^{1}$ \\ ${ }^{1}$ Department of Obstetrics, Gynecology and Reproductive Sciences, University of California, San Francisco, CA, USA E-mail: abcmd@berkeley.edu}

\section{References}

1 Menacker F, Curtin SC. Trends in cesarean birth and vaginal birth after previous cesarean, 1991-99. Natl Vital Stat Rep 2001; 49: 1-16.

2 Hamilton BE, Martin JA, Ventura SJ. Births: preliminary data for 2005. Health E-stats. Released November 21 2006, http://www.cdc.gov/nchs/products/pubs/ pubd/hestats/prelimbirths05/prelimbirths05.htm.
3 US Department of Health and Human Services. Office of Disease Prevention and Health Promotion - Healthy People 2010. Nasnewsletter 2000; 15: 3.

4 ACOG Committee opinion. Vaginal birth after previous cesarean birth. Number 143, October 1994. Committee on Obstetric Practice. American College of Obstetricians and Gynecologist. Int J Gynaecol Obstet 1995; 48(1): 127-129.

5 ACOG Committee Opinion. Mode of term singleton breech delivery. Number 340, July 2006. Committee on Obstetric Practice. American College of Obstetricians and Gynecologists. Obstet Gynecol 2006; 108(1): 235-237.

6 Hannah ME, Hannah WJ, Hewson SA, Hodnett ED, Saigal S, Willan AR. Planned cesarean section versus planned vaginal birth for breech presentation at term: A randomized multicentre trial. Term Breech Trial Collaborative Group. Lancet 2000; 56(9239): 1375-1383.

7 Whyte H, Hannah ME, Saigal S, Hannah WJ, Hewson J, Amankwah K et al. Outcomes of children at 2 years after planned cesarean birth versus planned vaginal birth for breech presentation at term: the international randomized term breech trial. Am J Obstet Gynecol 2004; 191(3): 864-871.

8 Caughey AB. Elective cesarean delivery: when is it justified? J Perinatol 2006; 26: $1-2$.

9 Angeja A, Washington AE, Vargas J, Gomez R, Rojas I, Caughey AB. Chilean women's preferences regarding mode of delivery: which do they prefer and why? Br J Obstet Gynaecol 2006; 113: 1253-1258. 